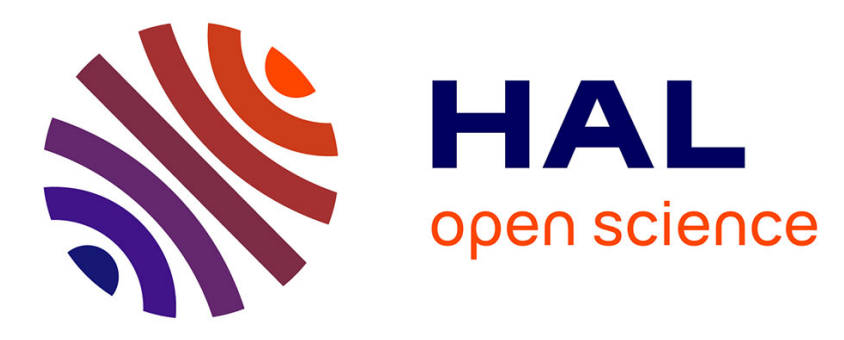

\title{
Study of the partial wetting morphology in polylactide/poly[(butylene adipate)-co-terephthalate]/polyamide ternary blends: case of composite droplets
}

\author{
Yang Fu, Gabriel Fodorean, Patrick Navard, Edith Peuvrel-Disdier
}

\section{To cite this version:}

Yang Fu, Gabriel Fodorean, Patrick Navard, Edith Peuvrel-Disdier. Study of the partial wetting morphology in polylactide/poly[(butylene adipate)-co-terephthalate]/polyamide ternary blends: case of composite droplets. Polymer international, 2018, 67 (10), pp.1378 - 1385. 10.1002/pi.5651 . hal01923291

\section{HAL Id: hal-01923291}

https://hal-mines-paristech.archives-ouvertes.fr/hal-01923291

Submitted on 15 Nov 2018

HAL is a multi-disciplinary open access archive for the deposit and dissemination of scientific research documents, whether they are published or not. The documents may come from teaching and research institutions in France or abroad, or from public or private research centers.
L'archive ouverte pluridisciplinaire HAL, est destinée au dépôt et à la diffusion de documents scientifiques de niveau recherche, publiés ou non, émanant des établissements d'enseignement et de recherche français ou étrangers, des laboratoires publics ou privés. 


\title{
Study of the partial wetting morphology in PLA/PBAT/PA ternary blends: Case of composite droplets
}

\author{
Yang FU ${ }^{1,2}$, Gabriel FODOREAN ${ }^{1,3}$, Patrick NAVARD ${ }^{1}$, Edith PEUVREL-DISDIER ${ }^{1}$ \\ ${ }^{1}$ MINES ParisTech, PSL Research University, CEMEF - Centre de mise en forme des matériaux, CNRS \\ UMR 7635, CS 10207 rue Claude Daunesse 06904 Sophia Antipolis Cedex, France \\ ${ }^{2}$ Present address : SABIC Europe, Urmonderbaan 22 Blg150, 6167 RD Geleen, The Netherlands \\ 3 Present address : Technical University of Cluj-Napoca, Faculty of Mechanical Engineering, \\ Department of Automotive Engineering and Transport, 103-105 Muncii Blvd., 400641, Cluj-Napoca, \\ Romania
}

Corresponding author : Edith Peuvrel-Disdier (edith.disdier@mines-paristech.fr)

Key-words: Ternary polymer blends, morphology, spreading coefficient, interfacial tension

\begin{abstract}
The prediction of the morphology of ternary polymer blends requires a good knowledge of the values of the three interfacial tensions. We selected three polymers, either biobased or biodegradable, PA, PBAT and PLA and we accurately measured their interfacial tensions using the retraction method, varying the molar mass or inverting the phases. The following values of interfacial tensions were obtained: $\gamma_{P B A T / P L A}=3.3 \pm 0.7 \mathrm{mN} / \mathrm{m}, \gamma_{P A / P L A}=5.6 \pm 0.3 \mathrm{mN} / \mathrm{m}$ and $\gamma_{P B A T / P A}=3.0 \pm 0.4 \mathrm{mN} / \mathrm{m}$. These values were used to calculate the spreading coefficients giving rise to two negative coefficients and one coefficient close to zero. Ternary blends with various compositions, two different levels of viscosity for the PBAT, different processing conditions were prepared. There was a very good agreement between the predictions of the spreading theory, when using the values of interfacial tensions in the right order of magnitude, and the observed morphologies, whatever the polymer serving as a matrix. When the PLA or PBAT was chosen as the matrix, the ternary blend morphology was composed of composite droplets, presenting a partial wetting morphology, dispersed in the polymer matrix. This morphology was observed whatever the composition, the viscosity level of the PBAT phase and the processing conditions. A further calculation of the free energy confirmed this
\end{abstract}


morphology. The formation process of this semi-encapsulated morphology was observed during blending.

\section{Introduction}

Blending different polymers in order to obtain controlled supramolecular structures/morphologies able to provide improved properties has been studied and applied long ago. ${ }^{1-4}$ Although several polymer pairs can be miscible ${ }^{5}$, most blends are formed by two, sometimes more, polymers which are phase separated in their fluid state. The obtained structures are then due to a combination of thermodynamic and kinetic aspects where the major parameters at stake are the interfacial tension between polymers (expressing the free energy barrier between them), the rheological properties of the individual polymers (mostly having an effect on the kinetics of morphology changes) and the processing conditions (controlling the morphology evolution and the final size). Binary blends can display a morphology composed of a matrix and a dispersed phase or a bi-continuous morphology.

Ternary blends can obviously display a much richer diversity of morphologies. Guo and co-workers ${ }^{6}$ were the first to use the expression of the free energy where they calculated $\sum A_{i j} \gamma_{i j}$ (where $A_{i j}$ stands for the interfacial area between the two phases, $\gamma_{i j}$ for their interfacial tension) for all possible morphologies of the same composition, allowing to predict the lowest free energy morphology at equilibrium. Another approach using the notion of spreading coefficient ${ }^{7}\left(\lambda_{i j}\right)$ was proposed by Hobbs and co-workers ${ }^{8}$ and further developed by the group of Favis ${ }^{9}$ considering the three spreading coefficients characterizing an $A / B / C$ ternary blend:

$$
\begin{aligned}
& \lambda_{B / A / C}=\gamma_{B C}-\gamma_{A B}-\gamma_{A C} \\
& \lambda_{A / B / C}=\gamma_{A C}-\gamma_{A B}-\gamma_{B C} \\
& \lambda_{A / C / B}=\gamma_{A B}-\gamma_{A C}-\gamma_{B C}
\end{aligned}
$$

Four possible morphologies can be described by combining the different signs for the three spreading coefficients, as shown in Figure 1. 
a.

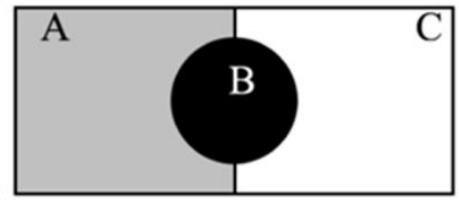

$\lambda_{B / A / C}<0$
$\lambda_{A / B / C}<0$
$\lambda_{A / C / B}<0$

b.

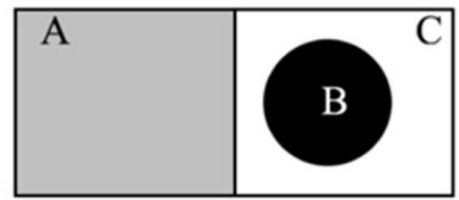

$\lambda_{B / A / C}<0$

$\lambda_{A / B / C}<0$

$\lambda_{A / C / B}>0$ c.

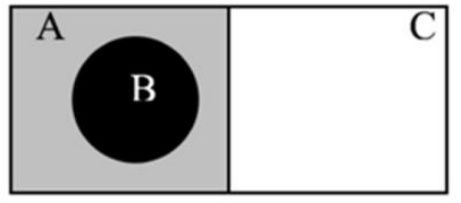

$\lambda_{B / A / C}>0$
$\lambda_{A / B / C}<0$
$\lambda_{A / C / B}<0$

d.

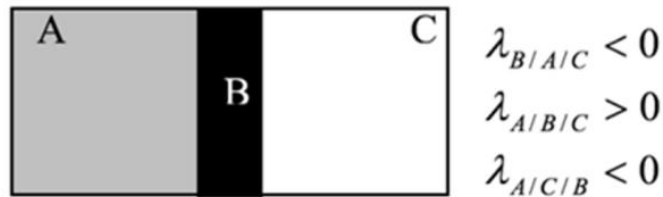

Figure 1: Four possible equilibrium morphologies for a ternary polymer blend, with a minor phase $C$ and two major phases $A$ and $B$, and their relations to the respective signs of the spreading coefficients, according to reference ${ }^{9}$.

The advantage of this approach is that the knowledge of the interfacial properties is enough to predict the morphology.

Favis and coworkers ${ }^{10}$ also proposed to take into account the contribution of the phase elasticity in the interfacial tension under shear and to consider this dynamic interfacial tension in order to estimate the minimum free energy of the blend.

In order to test the reliability of the models, various ternary blends were investigated to change of the interfacial tension balance and to obtain the different morphologies. ${ }^{11-24}$ The models derived from Guo and Hobbs have successfully predicted the morphologies in ternary blends in most situations ${ }^{12,24-25}$ where the large values of spreading coefficients or high contrast of free energy are the main factors that determine the type of morphology.

However, if the interfacial effect is weak (for example in particular in the case of partial wetting, Figure 1.a), these models may no longer provide a correct prediction. All factors which can play a role on the kinetics of the morphology development such as the viscosity, the elasticity, the compatibilization (reactive compatibilizers...), any reticulation process, the sequence of introduction may affect the obtained morphology. The viscosity ratio and the mixing parameters should better control the size of the domains.

A large attention has been devoted to the understanding of the physical origin and the characteristics of the core-shell morphology of composite droplets imbedded in another polymer matrix (Figures 1.b and 1.c). Studies have concerned the variety of structures which could be obtained in the composite droplets, ${ }^{26}$ the determination of the droplet size, of the relative thickness of the shell and their relative variation, ${ }^{26-29}$ their effect on the dynamic rheological behavior of the blend in comparison to the binary blend $d^{14,30}$ and of course the relationship with the mechanical properties..$^{17,20-23,31-34}$ 
More recently, a focus was given on partial wetting morphologies, ${ }^{9,35-36}$ where droplets of one polymer are located at the interface of the two other polymers (Figure 1.a), compared to a complete wetting of the interface by the third phase (Figure 1.d). The coarsening of these two types of morphologies in the case of co-continuous morphologies was investigated. ${ }^{37} \mathrm{~A}$ recent paper reported on the use of partially wetting droplets to compatibilize and toughen co-continuous ternary blends. ${ }^{38}$

Very few studies concern the mechanisms governing the evolution of the morphology. Reignier et al. ${ }^{11}$ showed the coalescence of core-shell droplets with time. Valera et al. ${ }^{13}$ showed the different steps leading to a core-shell morphology when the third polymer (dispersed phase) was introduced in the pre-mixed binary blend.

Interfacial tension thus plays a very important role in the control of the hierarchal morphology of ternary blends. This means that the measurement of this parameter is critical. Several methods have been proposed in order to measure the interfacial tension between two molten polymers. A possibility is to calculate it from surface tensions or solubility parameters. ${ }^{39}$ Another method classically used was proposed by $\mathrm{Wu}^{40}$ and relates the interfacial tension $\gamma_{i j}$ to the surface tension of two polymers $\gamma_{i}$ and $\gamma_{j}$ and to their polar and dispersive terms. The advantage of these two calculation methods is their apparent simplicity and the ease of measuring the needed parameters. However, due to their poor accuracy, they can only give a rough estimation of interfacial tensions. ${ }^{41}$

Another way is to balance the interfacial tension with another force which effect will be easy to measure, like in the thread breaking or drop retraction methods or methods based on the rheological response of the blend. ${ }^{40,42-44}$

The drop retraction method, used in this work, is based on the measurement of the kinetics of drop relaxation after the drop has been deformed by a flow in the form of an ellipsoid with axis $L$ and $D$. The deformability parameter of this ellipsoid is time dependent and defined as:

$$
D_{e f}=\frac{L-D}{L+D}
$$

Assuming that the only driving force for the drop retraction is the interfacial tension and in the case of a very small initial deformation, Taylor ${ }^{45}$ predicted that the deformability parameter has a decreasing exponential form (Equation 5 ) characterized by a drop relaxation time $\tau$ (Equation 6 ).

$$
D_{e f}=D_{e f} \exp \left(-\frac{t}{\tau}\right)
$$




$$
\tau=\frac{(2 p+3)(19 p+16)}{40(p+1)} \frac{\eta_{m} R_{0}}{\gamma_{A B}}
$$

$D_{e f_{0}}$ is the initial deformability parameter at $t=0 \mathrm{~s}$ of the considered relaxation process, $p$ is the viscosity ratio between the dispersed phase and the matrix, $R_{0}$ is the radius of the drop at the end of relaxation procedure, $\eta_{m}$ is the viscosity of the matrix and $\gamma_{A B}$ is the interfacial tension. The first measurement of the interfacial tension using this method was performed by Luciani. ${ }^{39}$ The limit of $^{\text {Tim }}$ validity of the model was evidenced experimentally by Assighaou et al. ${ }^{46}$

Measuring the interfacial tensions is not an easy task since the different methods are indirect, fraught with potential artefacts. As an example, selecting in the literature three polymer couples, it is possible to find a very wide range of interfacial tension values. For example, the interfacial tension of PMMA/PS is reported to be between 0.25 to $3.1 \mathrm{mN} / \mathrm{m},{ }^{10,18,47-53}$ the one of PA66/PS between 6.8 and $20 \mathrm{mN} / \mathrm{m}^{12,54-56}$ and the one of PLA/PBAT between 0.5 and $22 \mathrm{mN} / \mathrm{m}^{57-60}$ It is not always easy to understand the reasons leading to such large discrepancies. The problem is that no prediction of blend morphology can be made if the measured value-of the interfacial tension between the two polymers varies by a factor five to ten.

The aim of this work was thus to investigate the morphology of a ternary bio-based and biodegradable blends composed of poly(butylene adipate-co-terphthalate) (PBAT), polyamide (PA) and polylactide (PLA) of different compositions. The morphology prediction implied to measure with the best possible accuracy the three interfacial tensions. The drop retraction method was used exchanging the nature of the polymer forming the drop and varying the viscosity level of the PBAT in order to improve the accuracy of the measurements. Attention was paid to the partial wetting morphology observed for some compositions. We were interested in the blend composition range giving rise to composite droplets presenting a partial wetting morphology in complement to the cocontinuous morphologies studied by the group of Favis. ${ }^{47}$ The effect of different parameters such as, the blend composition, the exchange of the polymer matrix, the viscosity level of the PBAT phase and the processing parameters, on the blend morphology was investigated in order to test the reliability of the model prediction. A last part was devoted to the formation process of the partial wetting morphology during blending. 


\section{Experimental part}

\section{$\underline{\text { Materials }}$}

Polylactide (PLA 3051D) noted PLA from NatureWorks, Minnetonka, MN, USA, poly(butylene adipateco-terephthalate) (Ecoflex FBX 7011) noted PBAT from BASF, Ludwigshafen, Germany and a copolyamide (Plastamid HX2656), noted PA, from Arkema, Serquigny, France were chosen as components of the ternary blend due their respective immiscibility. Two batches of PBAT were investigated, $\mathrm{PBAT}_{1}$ (Newtonian viscosity of 3250 Pa.s at $180^{\circ} \mathrm{C}$ ) and $\mathrm{PBAT}_{2}$ (Newtonian viscosity of 150 Pa.s at $\left.180^{\circ} \mathrm{C}\right)$.

Polyesters and polyamide being sensitive to humidity, they were dried at $80^{\circ} \mathrm{C}$ in a vacuum oven for at least four hours prior to any use of the polymers in the molten state (compounding, compression molding and rheometry).

\section{Blend preparation}

The melt blending of the polymers was performed in two different mixing devices of different sizes. An internal mixer (Haake Rheocord 600P, ThermoFischer, Karlsruhe, Germany) was used to prepare $50 \mathrm{~g}$ of sample. The product from the internal mixer was then ground (grinder M50/80, Hellweg Maschinenbau GmbH \& Co KG, Roetgen, Germany) prior the next forming step. A micro-compounder (Haake Minilab II, ThermoFischer, Karlsruhe, Germany) was also used to prepare the blends using only an amount of $5 \mathrm{~g}$. It is equipped with two counter-rotating screws of $15 \mathrm{~mm}$ diameter. The screws constitute the mixing zone and they can be connected to a recirculation circuit including a parallelepiped tank allowing the matter to pass several times through the screws.

In both mixing pieces of equipment, the different components of the blend were introduced simultaneously in the mixer. Most blends were prepared in the internal mixer. The microcompounder was used to investigate the morphology developed in the ternary blends, since samples can be taken at various steps of the mixing process in an easy manner.

\section{$\underline{\text { Rheological measurements }}$}

Rheological measurements were performed with a controlled-strain rheometer (ARES, TA Instruments, Delaware, USA). All measurements were performed in a cone-and-plate geometry (25 $\mathrm{mm}$ diameter and a $0.04 \mathrm{rad}$ angle) at $180{ }^{\circ} \mathrm{C}$ under nitrogen environment. Disk-shape samples (thickness $1 \mathrm{~mm}$, diameter $25 \mathrm{~mm}$ ) were prepared by compression molding using a hot press (Carver M3853-0, Carver, Indiana, USA) at $180{ }^{\circ} \mathrm{C}, 25 \mathrm{MPa}$ for $8 \mathrm{~min}$. Angular frequency sweep 
measurements (from $10^{2}$ to $10^{-1} \mathrm{rad} / \mathrm{s}$ ) were performed in the linear domain of viscoelasticity of the neat components. Care was taken to check the thermal stability of the polymers and the linear domain prior to frequency sweep measurements.

Figure 2 shows the rheological behavior of the pristine polymers under small amplitude oscillatory shear.
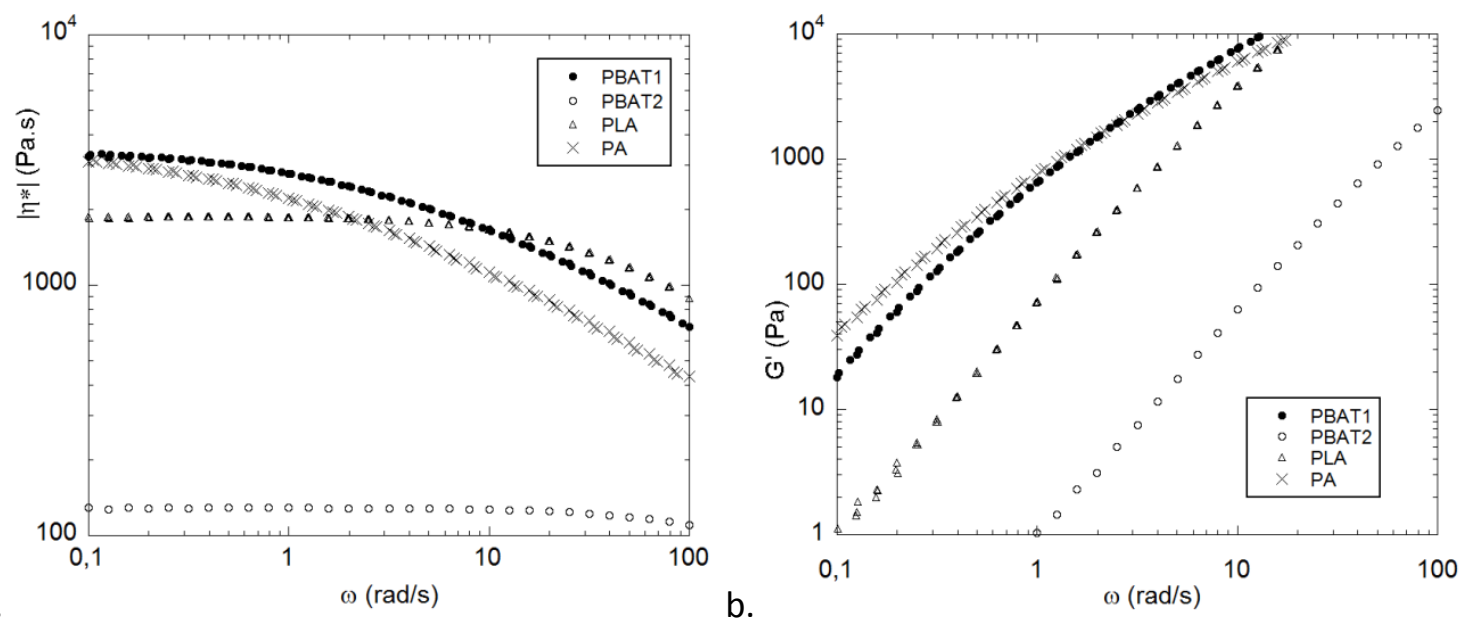

a.

b.

Figure 2: Small amplitude oscillatory shear data of the pristine polymers measured at $180^{\circ} \mathrm{C}$ : a. complex viscosity, b. storage modulus

The Newtonian viscosity for each polymer was estimated directly on Figure 2 or by fitting the viscosity curve with the Carreau-Yasuda model:

$$
\eta^{*}(\omega)=\eta_{0}\left[1+(\lambda \omega)^{a}\right]^{\frac{(m-1)}{a}}
$$

$\eta_{0}$ is the newtonian viscosity, $\lambda$ a characteristic relaxation time, $a$ the Yasuda parameter and $m$ the shear-thinning index

The zero shear viscosities are reported in Table 1.

\section{Scanning electronic microscopy (SEM)}

The morphologies of the blends were observed by scanning electronic microscopy (SEM, Philips XL 30 ESEM) using the back-scattered electron mode and an accelerating voltage around $15 \mathrm{kV}$. Observations were performed on cryo-fractured cross-sections of samples prepared for rheometry. Cross-section surfaces were systematically polished in order to obtain flat surfaces with a roughness parameter close to $1 \mu \mathrm{m}$ and then coated with $\mathrm{Au} / \mathrm{Pd}$ (deposit thickness around $10 \mathrm{~nm}$ ). This 
procedure made it possible to distinguish the different polymers even in the case of the ternary blends.

\section{$\underline{\text { Interfacial tension determination based on drop retraction }}$}

The drop retraction method implies the in-situ follow-up of the relaxation of a deformed drop. The deformation of the droplet was performed in a transparent counter-rotating shear cell coupled to a transmission optical microscope. The sample consists in a sandwich of a small piece of polymer A between two polymer films of the matrix polymer B. The sandwich sample was then introduced in the shear cell at $180^{\circ} \mathrm{C}$, molten, stabilized in temperature (after $5 \mathrm{~min}$ ) and squeezed to the nominal gap. The temperature was considered as homogeneous after $10 \mathrm{~min}$ of heating. The droplet phase imbedded in the matrix was deformed under the action of shear up to a certain deformation where the shear flow was stopped. The drop relaxation kinetics was monitored. The gap was fixed at 1000 $\mu \mathrm{m}$. The relaxation of 40 to $110 \mu \mathrm{m}$ radius drops was investigated. The relaxation kinetics of at least 6 to 10 drops per system was measured. Attention was paid to the following factors during these measurements. The temperature of the polymer in the transparent shear cell was controlled using 50 $\mu \mathrm{m}$ diameter thermocouples. Only a few degrees difference between different locations in the $4 \mathrm{~cm}$ diameter sample was allowed. The gap relatively to the drop dimension was large enough to ensure that the drop deformation or relaxation was not influenced by the wall vicinity. ${ }^{61}$ Droplets were slightly deformed to ensure that the relaxation only took place via the iso-volumetric retraction of the droplet toward the spherical shape. Relaxation data were only considered valid when the deformability parameter was small enough (typically starting from $\left.D_{e f} \sim 0.2\right) .{ }^{46}$ The measurement of the two dimensions (major and minor axis) of the projected droplet was preferred to the determination of the true length of the major axis from the volume conservation knowing the radius of the drop after retraction $\left(R_{0}\right)$ and the measurement of the minor axis. The deformation of the drop being very small, the interfacial tensions determined from direct measurements (projected length) or from corrected values were very close and within the error bars on these measurements. All values of interfacial tensions were determined from indirect measurements of the dimensions of the drops.

\section{Results and Discussions}

\section{Interfacial tension}

In order to compare morphologies to the theory based on the spreading coefficients, the interfacial tensions of the three couples must be known. A full description of the methodology followed to 
measure the interfacial tension of PLA/PBAT will be given in the following paragraph. The same procedure was followed for the two other couples but the results will be given without a full description.

\section{Interfacial tension of PLA/PBAT blend}

As mentioned in the introduction, very different values are reported on PLA/PBAT blends. ${ }^{57-60}$ The interfacial tension was measured by the drop retraction method. All experiments were conducted at $180^{\circ} \mathrm{C}$.

Measurements were performed on droplets of PLA in $\mathrm{PBAT}_{1}$ matrix, on the reverse system with droplets of PBAT 1 in PLA matrix, on droplets of PLA in the lower viscosity $\mathrm{PBAT}_{2}$ matrix and on the reverse system on droplets of $\mathrm{PBAT}_{2}$ in PLA matrix.

Figure 3 shows an example of a $\mathrm{PBAT}_{1}$ drop relaxation in PLA and Figure 4 depicts the time evolution of the drop deformability during the last relaxation stage plotted on a semi-log scale. The relaxation follows an exponential decay with a characteristic time (Equation 5 ) used to determine the interfacial tension (Equation 6).

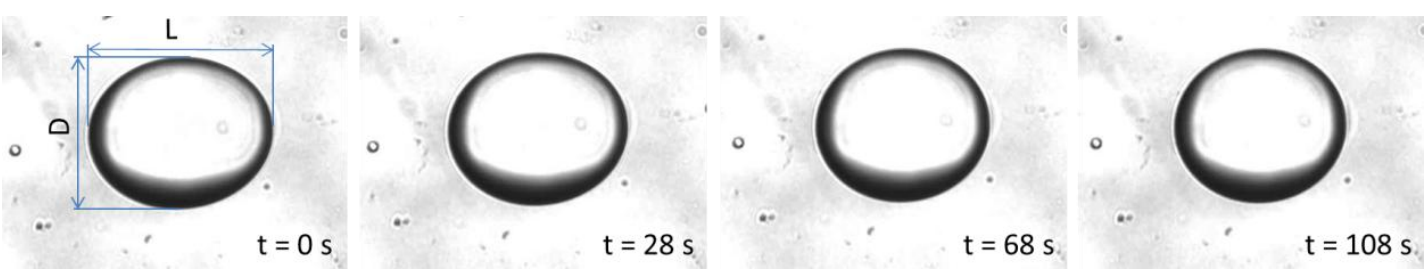

Figure 3: Relaxation of an ellipsoidal drop of $\mathrm{PBAT}_{1}$ in the PLA in-matrix after deformation under shear (flow direction is horizontal, vorticity axis is vertical). The drop radius is $83 \mu \mathrm{m}$.

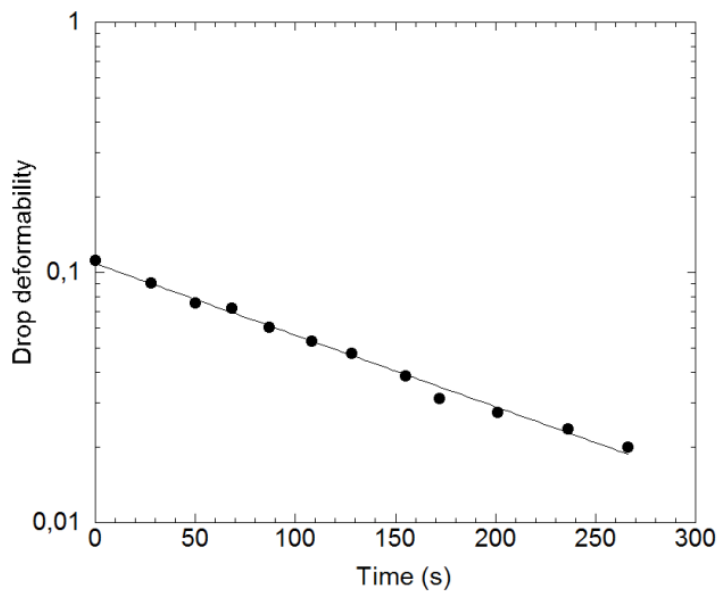

Figure 4: Time evolution of the deformability parameter in the case of Figure 3. 
Table 1 gives the different values of interfacial tensions for the four possible cases, i.e. PLA in PBAT 1 or $\mathrm{PBAT}_{2}$ and $\mathrm{PBAT}_{1}$ or $\mathrm{PBAT}_{2}$ in PLA. As expected, the results show that there is no effect of the molar mass of PBAT as well as no effect of which polymer is acting as the drop. The mean value of the PBAT/PLA interfacial tension, as averaged over all the obtained results, is $3.3 \pm 0.7 \mathrm{mN} / \mathrm{m}$.

\section{Interfacial tension of PA/PBAT and PA/PLA blends}

The fact that the PA copolymer was viscoelastic was considered to choose the polymer forming the droplet phase for the drop retraction experiments. Several authors investigated the effect of the viscoelastic character of the droplet or the matrix relatively to the case of two Newtonian fluids. ${ }^{62}$ The relaxation of a lowly deformed viscoelastic droplet imbedded in a Newtonian matrix was reported to follow an exponential decay with a relaxation time similar to a blend with two Newtonian phases, ${ }^{63}$ whereas a longer relaxation process was reported in the case of a Newtonian droplet in a viscoelastic fluid..$^{64}$ In order to limit the effect of viscoelasticity, the PA copolymer was chosen as the droplet phase. The retraction of PA droplets was measured in the PLA matrix and in both PBAT polymers.

When the drop retraction test was conducted on the PA drop in $\mathrm{PBAT}_{2}$, a different phenomenon was observed while deforming the PA drops: the drops did not deform along the shear direction as for the other cases but along the vorticity axis as shown on Figure 56 . The orientation of objects along the vorticity axis is a common feature which usually occurs when the dispersed phase is solid ${ }^{65}$ or fluid $^{66-68}$. When the dispersed phase is solid, orientation of elongated objects along the vorticity axis is due to the elasticity of the suspending medium. ${ }^{65}$ For fluid systems, such phenomena are taking place when the elasticity of the dispersed phase is much larger than the one of the matrix ${ }^{66-68}$ which is the case for PA drops in $\mathrm{PBAT}_{2}$. The direction of deformation has no influence on the retraction kinetics after cessation of flow and allows to measure the interfacial tension.

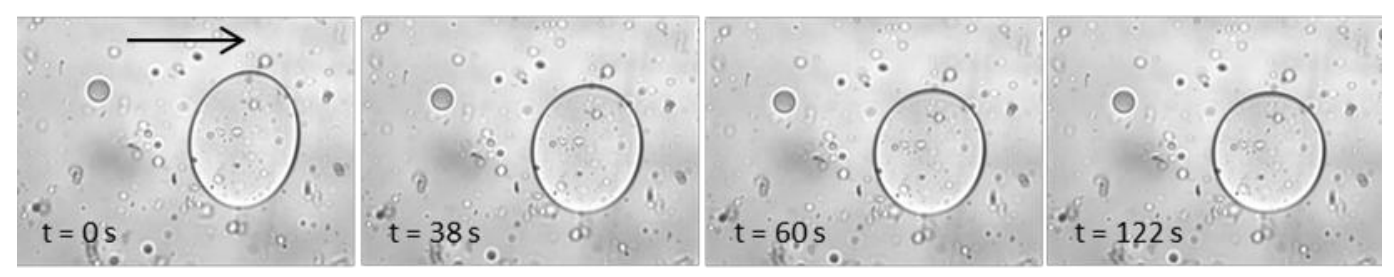

Figure 5: Retraction of PA drops in $\mathrm{PBAT}_{2}$ matrix after drop deformation. Deformation occurred along the vorticity axis (vertical), the flow direction being horizontal (indicated by the arrow). The radius of the drop is $106 \mu \mathrm{m}$.

The mean interfacial tension values based on the retraction method for the three polymer couples PLA/PBAT, PLA/PA and PBAT/PA are reported in Table 3. 


\section{Prediction of the morphology of ternary blends}

Using the values of the interfacial tensions given in Table 2, it is possible to calculate the values of the spreading coefficient:

$$
\begin{gathered}
\lambda_{P B A T / P A / P L A}=\gamma_{P B A T / P L A}-\gamma_{P A / P B A T}-\gamma_{P A / P L A}=-5.9 \pm 1.1 \mathrm{mN} / \mathrm{m} \\
\lambda_{P A / P B A T / P L A}=\gamma_{P A / P L A}-\gamma_{P B A T / P L A}-\gamma_{P A / P B A T}=-0.5 \pm 1 \mathrm{mN} / \mathrm{m} \\
\lambda_{P A / P L A / P B A T}=\gamma_{P A / P B A T}-\gamma_{P B A T / P L A}-\gamma_{P A / P L A}=-5.3 \pm 1.1 \mathrm{mN} / \mathrm{m}
\end{gathered}
$$

Considering error bars on the interfacial tension values, $\lambda_{P A / P B A T / P L A}$ appears slightly negative but could also be slightly positive, while the other two spreading coefficients are clearly negative. According to the spreading model, there are two possible morphologies as illustrated in Figure 1 which will depend whether $\lambda_{P A / P B A T / P L A}$ is above or below zero. When the three spreading coefficients are negative, which seems to be the case here, the PA drops are situated at the interface between the PLA and $\mathrm{PBAT}_{1}$ phases in a partial wetting situation (see in Figure 1.a). However, if $\lambda_{P A / P B A T / P L A}$ is above zero, then the PA droplets should be encapsulated by the PBAT phase (see Figure 1.d). To check if the spreading approach can predict the most probable morphology, two ternary blends of composition 60PLA/27PBAT/13PA were firstly prepared with the two PBAT polymers and examined by SEM. Figure 6 shows an example of morphology in the case of PBAT 1 grade. The black inclusions standing for the phase PA are mainly located at the interface between the dark gray phase $\left(\mathrm{PBAT}_{1}\right)$ and the white PLA phase. This is in agreement with the partial wetting morphology predicted by the spreading coefficients with all three negative coefficients. PA phase seems to have a preferential affinity with the PBAT phase, sign that $\lambda_{P A / P B A T / P L A}$ is close to zero. Similar partial wetting morphologies were also found with the $\mathrm{PBAT}_{2}$ grade. This was surprising since the PBAT 2 had a much lower viscosity than the PBAT 1 . The factor $\frac{\eta_{P B A T}}{\eta_{P A}} \frac{\Phi_{P A}}{\Phi_{P B A T}}$ was estimated for both PBAT grades in order to determine which phase should be the matrix in the composite droplet. ${ }^{69}$ The viscosity values were considered at a mean shear rate of $25 \mathrm{~s}^{-1}$ corresponding to the condition 50 rpm in the internal mixer. Values smaller than one were obtained in all cases, confirming that PBAT is the matrix for the investigated range of volume fraction and whatever the viscosity level of the PBAT phase. 


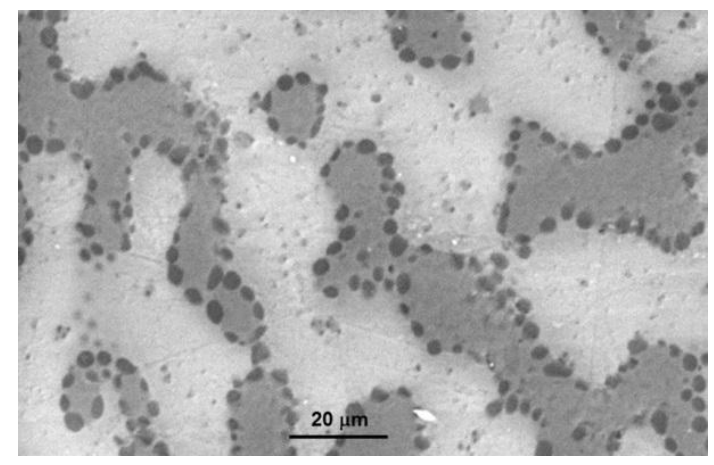

Figure 6: SEM micrography of a 60PLA/27PBAT 1 /13PA ternary blend prepared in the internal mixer: at $180^{\circ} \mathrm{C}, 50 \mathrm{rpm}$ for $12 \mathrm{~min}$. The black phase is $\mathrm{PA}$, the dark gray phase is $\mathrm{PBAT}_{1}$ and the light gray phase is PLA.

Blends with other compositions where the matrix is varied (60PLA/20PBAT $2 / 20 \mathrm{PA}$, $60 \mathrm{PBAT}_{2} / 20 \mathrm{PA} / 20 \mathrm{PLA}$ and 60PA/20PLA/20PBAT 2 ) were also prepared in order to further check the validity of the spreading coefficient theory. The three blends were prepared in the internal mixer in the same conditions $\left(180^{\circ} \mathrm{C}, 80 \mathrm{rpm}\right.$ for $\left.12 \mathrm{~min}\right)$. The experimental morphologies are shown in Figure 7.a to $\mathrm{c}$ and the corresponding predictions of morphology based on the spreading coefficient theory in Figure 7.d to $\mathrm{f}$. This figure shows that the experimental morphologies are matching the predictions.

a.
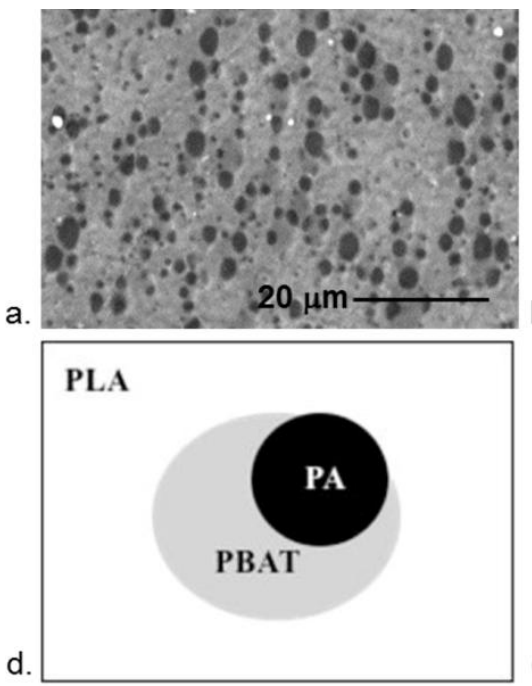

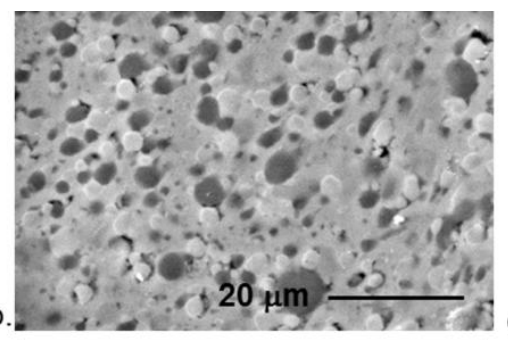

PBAT

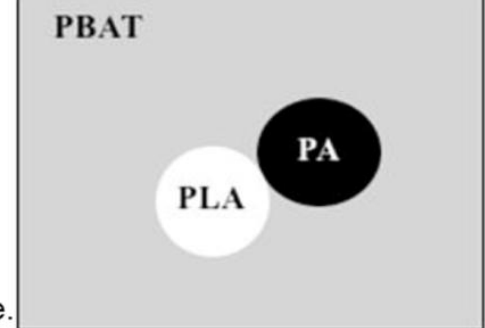

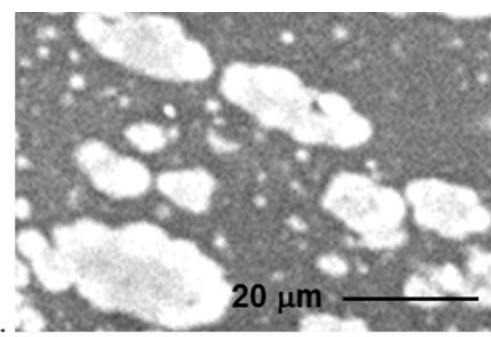

PA

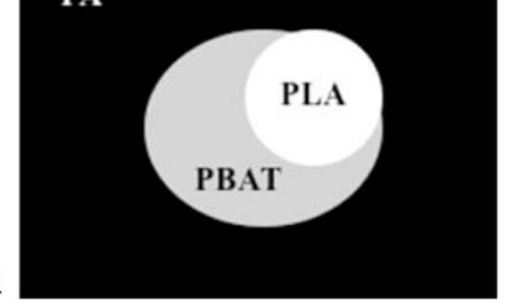

Figure 7: Morphologies of the three blends: a. 60PLA/20PBAT $2 / 20 \mathrm{PA} ;$ b. $60 \mathrm{PBAT}_{2} / 20 \mathrm{PA} / 20 \mathrm{PLA}$ and c. 60PA/20PLA/20PBAT 2 as seen by SEM and their respective model predictions (d. to f.). Blends were prepared in the internal mixer at $180^{\circ} \mathrm{C}, 80 \mathrm{rpm}$ for $12 \mathrm{~min}$. 
The effect of the mixing conditions was further investigated in the case of the 60PLA/2OPBAT $2 / 20 \mathrm{PA}$ blend by varying the rotation speed (30 to $100 \mathrm{rpm}$ ), the temperature (170 to $190^{\circ} \mathrm{C}$ ) or the mixing time ( 8 to $20 \mathrm{~min}$ ). The type of morphology was in all cases well predicted by the spreading model.

The spreading theory is correctly predicting the observed morphologies of the PLA/PBAT/PA ternary polymer blends. This is in agreement with other works ${ }^{24-25,27}$

We found a good agreement between the morphologies of the ternary blends based on PLA, PBAT and PA, using the interfacial tensions that we tried to measure with accuracy. Since a large range of interfacial tensions is reported in the literature for the polymer couple PLA/PBAT, one can wonder whether considering other values of interfacial tensions for this couple of polymers would lead the same prediction of the morphology. Considering the interfacial tensions of PA/PLA and of PA/PBAT as constant, it is interesting to note that an interfacial tension for PLA/PBAT between 2.6 and $8.6 \mathrm{mN} / \mathrm{m}$ would lead to the prediction of a partial wetting morphology (all spreading coefficients being negative), whereas an interfacial tension lower than 2.6 or larger than 8.6 would lead to an encapsulated morphology (one spreading coefficient being positive with encapsulation of PLA in

PBAT phase if $\gamma_{P B A T / P L A} \leq .2 .6 \mathrm{mN} / \mathrm{m}$ and encapsulation of PLA in PA phase if $\gamma_{P B A T / P L A} \geq .8 .6$ $\mathrm{mN} / \mathrm{m}$ ). This means that interfacial tensions need to be determined with care in order to be in the right order of magnitude. The good agreement between the different morphologies of the ternary blends obtained while exchanging the matrix and the spreading coefficient theory confirms that the interfacial tensions which were measured are in the right order of magnitude.

\section{Interpretation of the morphology based on thermodynamic aspects}

As mentioned, the morphology of the ternary blend 60PLA/27PBAT 1 /13PA shown on Figure 6 is a partial wetting one. PA is forming droplets which are scattered all over the interface between PBAT and PLA. This is the situation represented on Figure 8A. 

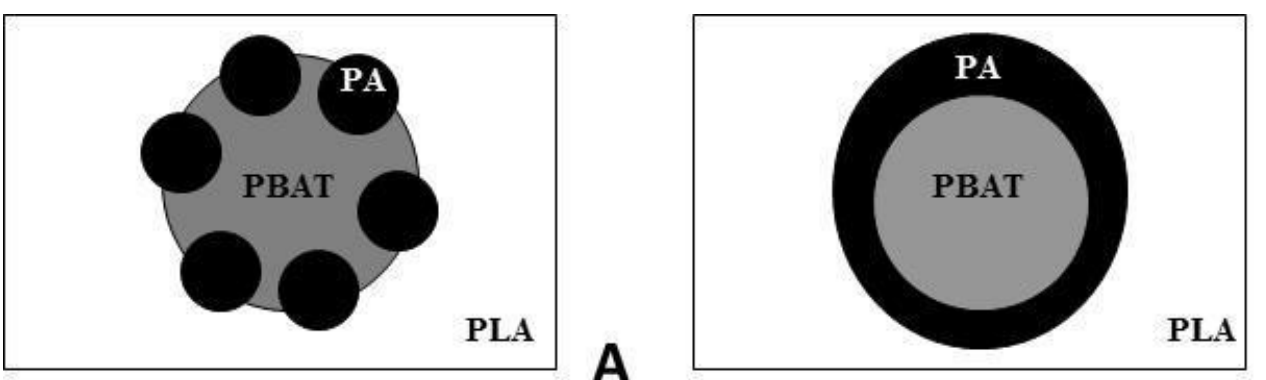

B

Figure 8: Two possible morphologies for the 60PLA/20PBAT/20PA blends.

An alternating morphology, PBAT encapsulated by a continuous outer layer of PA (full wetting), was tested. The question was to know why the PA phase could not form a stable layer around PBAT, as depicted on Figure 8B. Although the spreading theory was showing that the morphology A (Figure 8A and Figure 1.a) was the most favorable one, the calculation of the free energy of the morphologies for these two cases was performed. According to Guo and co-workers ${ }^{6}$, the free energy is $\sum A_{i j} \gamma_{i j}$ (where $A_{i j}$ stands for the interfacial area between two phases, $\gamma_{i j}$ for their interfacial tension), considering that the contribution of the partial molar free energy is the same for these two cases since they have the same composition. It is thus possible to compute the free energy of these two cases $A$ and $B$ :

$$
\begin{gathered}
E_{A}=4 \pi R_{P B A T}^{2}\left(\left(\frac{k+2}{2}\right)^{2 / 3} \gamma_{P L A / P B A T}+\frac{1}{4} k^{2 / 3} n^{2 / 3}\left(2 \gamma_{P A / P B A T}+2 \gamma_{P A / P L A}-\gamma_{P L A / P B A T}\right)\right) \\
E_{A}=4 \pi R_{P B A T}^{2}\left(\gamma_{P A / P B A T}+(k+1)^{2 / 3} \gamma_{P A / P L A}\right)
\end{gathered}
$$

where $R_{P B A T}$ is the radius of PBAT drops (same in both cases), $n$ is the number of PBAT drops and $k$ is the volume ratio between PA and PBAT. Assuming that $n=10$, the relative value of $E_{A}$ and $E_{B}$ are:

$$
\begin{gathered}
E_{A}=8.5 \times 4 \pi R_{P B A T}^{2} \\
E_{A B}=10.5 \times 4 \pi R_{P B A T}^{2}
\end{gathered}
$$

The result shows that the free energy of morphology A is lower than that of $B$. It confirms that morphology A representing a partial wetting morphology is also the more favorable one from the thermodynamic point of view for this polymer system.

\section{Dynamic aspects of morphology development}


In order to see how the blend morphology was transformed from the three polymers macroscopically separated ( $\mathrm{mm}$ range) into a more intimate structure (shown on Figure 6), samples were collected during the mixing operation (dead stop experiments) in the recirculation zone of the micro-compounder (transitional zone between two successive passages of the blend in the twin screw zone). The reservoir being larger than the zone driving the mix, there is a sudden enlargement of the lateral dimension and thus the establishment of an extensional flow perpendicular to the flow direction. Samples were collected in this diverging zone. Figure 9.a shows a "tree-like" morphology which is generated in the elongational field at the entrance of the recirculation reservoir for a 60PLA/20PBAT 1 /20PA blend.

a.

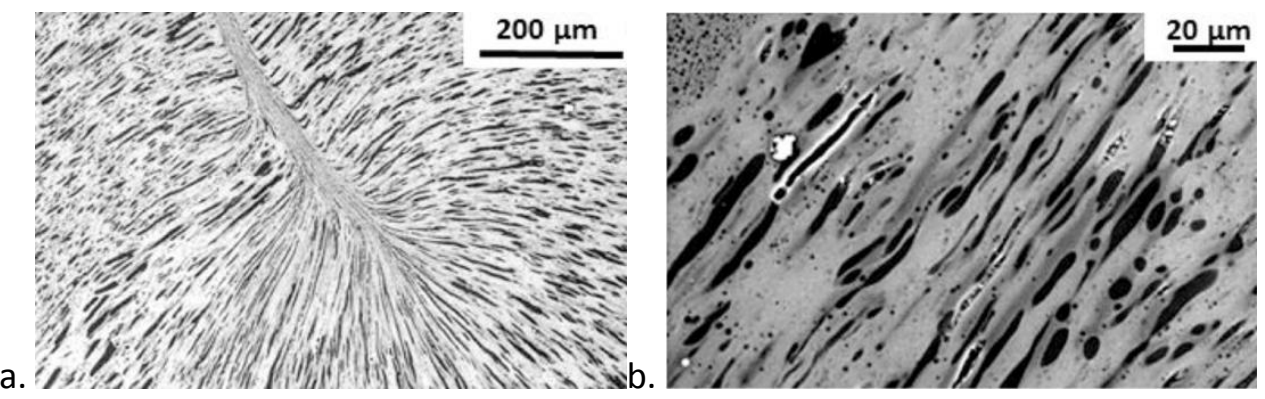

Figure 9: Morphology pattern of the ternary blend 60PLA/20PBAT $1 / 20 \mathrm{PA}$ at the diverging entrance of the recirculating reservoir in the micro-compounder $\left(180^{\circ} \mathrm{C}, 80 \mathrm{rpm}, 12 \mathrm{~min}\right)$ : a. overall view; $\mathrm{b}$. enlargement of a zone showing the twinning of PA (black) and PBAT (dark gray) phases.

The visual aspect of the tree-like pattern at the entrance of the reservoir is due to the PBAT/PA inclusions elongated along the flow direction. A closer look at this morphology shows that every branch of this tree-like pattern is composed by PBAT and PA phases (Figure 9.b) due to their affinity (lowest interfacial tension of the three couples, Table 2). In the complex flux introduced by the micro-compounder, once a PA inclusion meets a PBAT inclusion, they are staying together. Then the elongated PA threads break at the interface of PLA and PBAT by rupture mechanism or Rayleigh instabilities depending on flow rate and time. In some favorable situations, this last mechanism should give a very regular distribution of PA droplets at the interface between PLA and PBAT phases, as reported by Ravati et al. ${ }^{35,37}$.

\section{Conclusion}

A first part of this paper was dedicated to the determination of interfacial tensions using the drop retraction method varying the molar mass of the PBAT and inverting the phases. No effect of the 
molar mass was detected. Inverting the phases had no effect on the interfacial tension. The interfacial tensions between the three polymer couples are $\gamma_{P B A T / P L A}=3.3 \pm 0.7 \mathrm{mN} / \mathrm{m}, \gamma_{P A / P L A}=$ $5.6 \pm 0.3 \mathrm{mN} / \mathrm{m}$ and $\gamma_{P B A T / P A}=3.0 \pm 0.4 \mathrm{mN} / \mathrm{m}$. Based on this, spreading coefficients were calculated, giving $\lambda_{\mathrm{PBAT} / \mathrm{PA} / \mathrm{PLA}}=-5.9 \mathrm{mN} / \mathrm{m}, \lambda_{\mathrm{PA} / \mathrm{PBAT} / \mathrm{PLA}}=-0.5 \mathrm{mN} / \mathrm{m}, \lambda_{\mathrm{PA} / \mathrm{PlA} / \mathrm{PBAT}}=-5.3 \mathrm{mN} / \mathrm{m}$, with one of them being very close to zero. Polymer blends composed of bio-based PLA and PA and biodegradable PBAT were prepared varying the composition, the processing parameters, the PBAT viscosity level and exchanging the matrix (60PLA/27PBAT/13PA, 60PBAT/2OPA/20PLA, 60PA/20PLA/20PBAT). Different morphologies were obtained which were in agreement with the prediction of the spreading coefficient theory. This shows that the determined interfacial tensions were at least in the right order of magnitude. The sensitivity of the morphology to the variation of the interfacial tension of PBAT/PLA, while keeping the two others constant, was examined. In fact the interfacial tension needs to be in a certain range of values which is quite large.

In blends where the PLA or the PBAT was the matrix, a partial wetting morphology was obtained, sometimes with a very regular distribution of drops of nearly the same size along the interface of the two other polymers. In this case of partial wetting morphology, a free energy calculation confirms its occurrence, compared to the case of a complete wetting where a thin layer of one polymer is covering the interface of the droplet formed by the other phase. The type of morphology was in all cases determined by the interfacial tension balance. This was also true in the case of the partial wetting morphology, even when the PBAT viscosity level was much lower than the other phases.

Observations of the process formation of composite droplets with a partial wetting morphology tend to show that this morphology results from the elongation and rupture of coupled filaments of PBAT and PA.

Provided that a reliable measurement of interfacial tensions is made, this set of results, added to the several other ones already published, shows that morphologies of ternary blends can be reasonably theoretically predicted, at least when no kinetic effect is slowing down the formation of the thermodynamically stable structure.

\section{Acknowledgements}

This work was performed in the frame of the Industrial Chair on Bioplastics supported by Arkema, L'Oréal, Nestlé, PSA and Schneider Electric. 


\section{References}

1 Paul DR and Newman S, Polymer Blends, volume 1, Academic Press, New York (1978).

2 Martuscelli E, Palumbo R and Marian Krysewski M, Polymer Blends: Processing, Morphology, and Properties, volume 2, Springer, Plenum Press, New York (1980).

3 Paul DR and Bucknall CB, Polymer blends: Formulation in Polymer blends: Formulation, Volume 1, Wiley Interscience, New York (2000).

4 Thomas S, Grohens $\mathrm{Y}$ and Jyotishkumar P, Characterization of Polymer Blends: Miscibility, Morphology and Interfaces, Wiley, NewYork (2014).

5 Coleman MM, Painter PC and Graf JF, Specific Interactions and the Miscibility of Polymer Blends, Technomic Publishing Company, Lancaster, USA (1991).

6 Guo HF, Packirisamy S, Gvozdic NV and Meier DJ, Polymer 38: 785-794 (1997).

7 Harkins WD, J Chem Phys 9: 552-568 (1941).

8 Hobbs SY, Dekkers M and Watkins VH, Polymer 29: 1598-1603 (1988).

9 Le Corroller P and Favis BD, Polymer 52: 3827-3834 (2011).

10 Reignier J, Favis BD, and Heuzey MC, Polymer 44: 49-59 (2003).

11 Reignier J and Favis BD, Macromolecules 33: 6998-7008 (2000).

12 Omonov TS, Harrats C and Groeninckx G, Polymer 46: 12322-12336 (2005).

13 Valera TS, Morita AT and NR Demarquette, Macromolecules 39: 2663-2675 (2006).

14 Mohammdigoushki H, Nazodast H and Mostofi N, J Elastomers Plast 41: 339-351 (2009).

15 Virgillio N, Desjardins P, L’Espérance and Favis BD, Macromolecules 42: 7518-7529 (2009).

16 Abolhasani HM, Arefazar A and Mozdianfard M, J Polym Sci: Part B 48: 251-259 (2010).

17 Liu H, Song W, Chen F, Guo L and Zhang J, Mocromolecules 44: 1513-1522 (2011).

18 Kanzawa T and Tokumitzu K, J Appl Polym Sci 121: 2908-2918 (2011).

19 Saeb MR, Khonakdar HA, Razban SH, Garmabi H and Wagenknecht U, Macromol Chem Phys 213: 1791-1802 (2012). 
20 Li LP, Yin B, Zhou Y, Gong L, Yang MB, Xie BH and Chen C, Polymer 53: 3043-3051 (2012).

21 Khalili R, Jafari SH, Saeb MR, Khonakdar HA, Wagenknecht U and Heinrich G, Macromol Mater Eng 299: 1197-1212 (2014).

22 Jazani OM, Goodarzi V, Hemmati F and Saeb MR, J Polym Res 23: 231, 14p (2016)

23 Li H and Xie XM, Polymer 108: 1-10 (2017)

24 Shokoohi S and Arefazar A, Polymers for Advanced Technologies 20: 433-447 (2009).

25 Wilkinson AN, Clemens ML and Harding VM, Polymer 45: 5239-5249 (2004).

26 Reignier J and Favis BD, AlChE J 49: 1014-1023 (2003).

27 Tchomakov KP, Favis BD, Huneault MA and Champagne MF, Polym Eng Sci 44: 749-759 (2004).

28 Saeb MR, Khonakdar HA, Razban M, Jafari SH, Garmabi and Wagenknecht U, Macromol Chem Phys 213 :1791-1802 (2012).

29 Rastin H, Jafari SH, Saeb MR, Khonakdar HA, Wagenknecht U and Heinrich G, Eur Polym J 53 :112 (2014).

30 Fahrländer M, Friedrich C, Rheol Acta 38: 206-2013 (1999).

31 Jazani OM, Arefazar A, Jafari SH ans Saeb MR, J Polym Eng Sci 31: 237-241 (2011).

32 Saeb MR, Khonakdar HA, Jafari SH, Jalifar N, Razban M and Wagenknecht U , Polym Bull 71 :613624 (2014)

33 Rastin H, Jafari SH, Saeb MR, Khonakdar HA, Wagenknecht U and Heinrich G, J Polym Res 21 :352 (2014).

34 Rastin H, Saeb MR, Jafari SH, Khonakdar HA, Kritzschmar B and Wagenknecht U, Macrom Mater Eng 300 :86-98 (2015).

35 Ravati S and Favis BD, Polymer 54: 3271-3281 (2013).

36 Zolali AM and Favis BD, J Phys Chem B 120: 12708-12719 (2016).

37 Ravati S and Favis BD, Polymer 54: 6739-6751 (2013).

38 Zolali AM and favis BD, Polymer 114: 277-288 (2017). 
39 Luciani A, Champagne MF and Utracki LA, J Polym Sci Part B: Polym Phys 35: 1393-1403 (1997).

40 Wu S, Polym Eng Sci 27: 335-343 (1987).

41 Biresaw G and Carriere CJ, J Polym Sci Part B: Polym Phys 40: 2248-2258 (2002).

42 Elemans P, Janssen J and Meijer H, J Rheol 34: 1311-1325 (1990).

43 Palierne JF, Rheol Acta 29: 204-214 (1990).

44 Bousmina M, Rheol Acta 38: 73-83 (1990).

45 Taylor GI, Proc Royal Soc A: Math, Phys Eng Sci 138: 41-48 (1932).

46 Assighaou S, Benyahia L, Rheol Acta 49: 677-686 (2010).

47 Carriere CJ, Cohen A and Arends CB, J Rheol 33: 681-689 (1989).

48 Cohen A and Carriere CJ, Rheol Acta 28: 223-232 (1989).

49 Elmendorp JJ, A Study on Polymer Blending Microrheology. PhD thesis, Delft University of Technology (1986).

50 Friedrich C, Gleinser W, Korat E, Maier D and Weese J, J Rheol 39: 1411-1425 (1995).

51 Gramespacher H and Meissner J, J Rheol 36: 1127-1141 (1992).

52 Joseph DD, Arney MS, Gillberg G, Hu H, Hultman D, Verdier C and Vinagre TM, J Rheol 36: 621662 (1992).

53 Rundqvist T, Cohen A and Klason C, Rheol Acta 35: 458-469 (1996).

54 Cho K, Jeon HK, Park CE, Kim J and Kim KU, Polymer 37: 1117-1122 (1996).

55 Son Y, Polymer 42: 1287-1291 (2001).

56 Xing P, Bousmina M, Rodrigue D and Kamal MR, Macromolecules 33: 8020-8034 (2000).

57 Al-Itry R, Lamnawar K and Maazouz A, Rheol. Acta 53: 501-517 (2004).

58 Jajali Dil E, Carreau PJ and Favis BD, Polymer 68: 202-2012 (2015).

59 Nofar M, Maani A, Sjoudi H, Heuzey MC, Carreau PJ, J Rheol 59: 317-333 (2015).

60 Lu X, Zhao X, Yang X and Xiao P, Polym Test 60 58-67 (2017). 
61 Bikard J, Menard P, Budtova T, Peuvrel-Disdier E, Comput Mater Sci 37: 517-525 (2006).

62 Guido S, Current Opinion in Colloid and Interf 16: 61-70 (2011).

63 Cardenaels et al, Rheol Acta 49: 941-945 (2010).

64 Sibillo V, Simeone M, Guido S, Greco F, Maffetone PL, J Non-Newt Fluid Mech 134: 27-32 (2006)

65 Dubois J, Fyen W, Rusu D, Peuvrel-Disdier E and Navard P, J Polym Sci, Polym Phys Ed 36: 20052013 (1998).

66 Zanina A and Budtova T, Macromolecules 35: 1973-1975 (2002).

67 Miglera KB, J Rheol 44: 277-290 (2000).

68 Mighri F and Huneault MA, J Appl Polym Sci 100: 2582-2591 (2006).

69 Miles IS and Zurek A, Polym Eng Sci 28: 796-805 (1988). 


\section{Tables:}

Table 1: Newtonian viscosity at $180^{\circ} \mathrm{C}$ of the pristine polymers using the Carreau-Yasuda model

\begin{tabular}{|l|l|l|l|l|}
\hline$\underline{\text { Polymer }}$ & $\underline{\text { PBAT }_{1}}$ & $\underline{\text { PBAT }_{2}}$ & $\underline{\text { PLA }}$ & $\underline{\text { PA }}$ \\
\hline$\underline{\eta_{0} \text { (Pa.s) }}$ & $\underline{3250}$ & $\underline{150}$ & $\underline{1800}$ & $\underline{3300}$ \\
\hline
\end{tabular}

Table 2: Mean interfacial tensions of the PLA/PBAT couple as measured by the drop retraction method varying the molar mass of PBAT and inverting which polymer is the drop.

\begin{tabular}{|l|l|l|}
\hline & PLA in PBAT & PBAT in PLA \\
\hline PBAT $_{1}$ & $3.4 \pm 0.8 \mathrm{mN} / \mathrm{m}$ & $3.3 \pm 0.7 \mathrm{mN} / \mathrm{m}$ \\
\hline PBAT $_{2}$ & $3.6 \pm 0.8 \mathrm{mN} / \mathrm{m}$ & $3.0 \pm 0.4 \mathrm{mN} / \mathrm{m}$ \\
\hline
\end{tabular}

Table 3: Mean interfacial tension values of the three couples PLA/PBAT, PLA/PA and PBAT/PA

\begin{tabular}{|l|l|}
\hline & Interfacial tension \\
\hline PA/PLA & $5.6 \pm 0.3 \mathrm{mN} / \mathrm{m}$ \\
\hline PLA/PBAT & $3.3 \pm 0.7 \mathrm{mN} / \mathrm{m}$ \\
\hline PBAT/PA & $3.0 \pm 0.4 \mathrm{mN} / \mathrm{m}$ \\
\hline
\end{tabular}

